\title{
The Application of Cellular Automaton in the Barrier Tolls
}

\author{
Xiangyu Liu \\ School of North China Electric Power University Baoding, Baoding 071000, China
}

18730272577@163.com

Keywords: Cellular Automaton, queuing model, barrier tolls, ETC

\begin{abstract}
Based on Cellular Automaton and Monte Carlo method, this paper constructs a model to discuss the problem of merging after toll, and redesigns the shape, size, and merging pattern of the toll area. Firstly, the movement of the vehicle is broken down into three stages to establish the corresponding sub-models: $\mathrm{M}$ / M / $\mathrm{n}$ queuing model, vehicle-following model as well as merging model. Then a series of indicators are set up to analyze the toll plaza, such as Danger Index and Efficiency Index. Afterwards, the CA is taken to simulate traffic flow and the result of simulation is listed in the table 1. By changing the area of entrance and exit, we can draw a conclusion that: safety and efficiency are always negatively related to costs. With the increase of area, the throughput and Efficiency Index will increase at the same time while Danger Index decreases by contraries.
\end{abstract}

\section{Introduction}

With the continuous development of highways, highway traffic accident rate is also increased every year. Toll system is an important part of the highway. Currently, the "barrier tolls" is one of the common highway toll charging methods. A barrier toll is a row of tollbooths placed across the highway, and there are usually more tollbooths than there are incoming lanes of traffic. Therefore, when the car leaves the toll station, the traffic must be fan-shaped from the exit of the wider toll station into the less conventional lane of the lane ${ }^{[1]}$. The toll plaza was created to improve the congestion after the card, including the multi-lane area before the toll station, the toll booth itself, and the fan-in area after the toll gate. The construction of toll plaza has become one of the difficult problems of government and relevant departments.

Previously, a large number of traffic simulations were based on Cellular Automata (CA), indicating that the CA model is a feasible and effective method to emulate traffic flow. Space, time, and all state are discrete in Cellular Automata. For instance, the model divides the road into small rectangular cells and divides the time into multiple small cells. This feature greatly simplifies the simulation process. In addition, the state surface is controlled by adjacent cells following a set of rules, which is very similar to real-life traffic, where the movement of the car is greatly dependent on the adjacent cars' movement. Therefore, we apply Cellular Automata to solve our problem is reasonable.

In our simulations, we divide each channel into one thousand cells. The length and width of each cell is 4 meters, and the cell is empty when no vehicle is running. For the sake of simplicity, we only consider one direction of the freeway. Thus, $n$-way freeways are converted to $n \times 1000 n \times 1000$ matrices.

In our simulation, for the sake of simplicity, we ignore the vehicle speed, volume and other factors. All cars are considered the same species. For each lane, the first 6 cells were used as car-producing regions, throughput was observed in the last 10 cells, and transport density was calculated based on the last 500 cells. Our model is updated once per second, while $T=1 \mathrm{~s}$ is the average response time of the drive.

\section{Design of Cellular Automata ${ }^{[2]}$}

We discuss the basic process of the CA model:

- Inflow Process: According to the inflow model that we will discuss in a moment, assign vehicles 
in the vehicle-generation area.

- Acceleration Process: If $V<V m$, a vehicle accelerates by $\Delta V$, and the new speed is $V^{\prime}=V+\Delta V$.

- Deceleration Process: If the distance between a vehicle and the vehicle ahead of it (Front bumper to front bumper distance, we call it the gap, and the gap is denoted by $G$ and its unit is cell. When there is no vehicle ahead, $G$ is set to $+\infty$ ) is no more than $V$, the vehicle decelerates to $V^{\prime}=(G-1) / T$.

- Moving Process: Vehicles move forward by $V^{\prime *} T$ cells only when $G>G_{s}\left(V^{\prime}\right) . G_{s}\left(V^{\prime}\right)$ is the minimum gap required for safety consideration, and is to be defined later.

The flowing model can be used to simulate the process of vehicles passing through toll stations. For each lane, the first six cells in the Cellular Automata are set as vehicle generation area. We assume that the arrival of each vehicle obeys the Poisson probability distribution.

$$
P_{t}(N)=\frac{\lambda^{N}}{N !} e^{-\lambda}, N>0
$$

The time to generate the number of vehicles per one simulation is $t$ second through the Poisson distribution. The value of $t$ as a second. $\mathrm{N}$ represents the total number of vehicles arriving every second. The smaller the $\lambda$ is, the lighter the traffic is; the greater, the heavier. Therefore, we simulate the different traffic conditions by assigning the corresponding $\lambda$ values.

\subsection{Queuing model ${ }^{[3]}$}

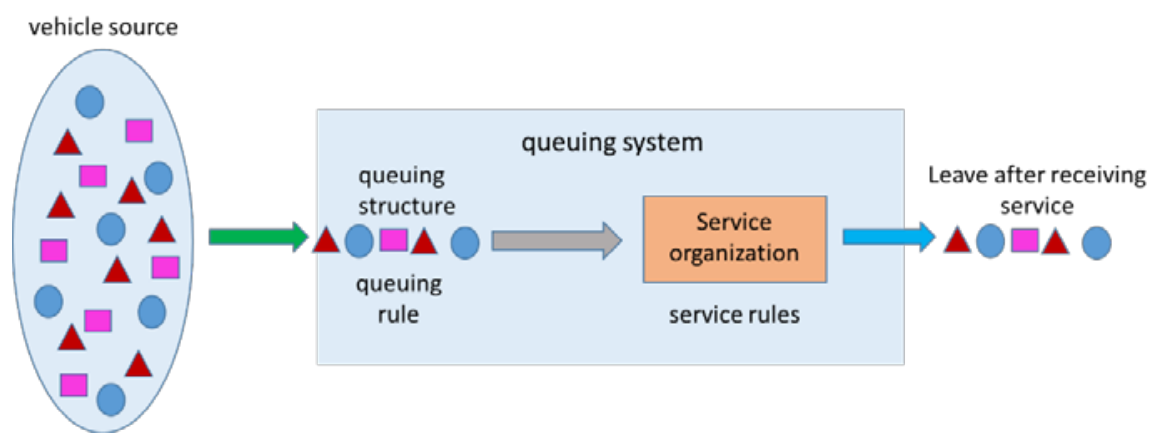

Fig1. Queuing model diagram

Vehicles on the expressway to reach the toll station, waiting in line, followed by charging service, and then leave the toll station. If the arrival of vehicles cannot get services in time, resulting in a queuing phenomenon. The highway toll system is a typical queuing system. The toll station system adopts multi-channel and multi-service, which is a standard $P / M / B / \infty / \infty / F C F S$ queuing theory mode.

\subsection{Vehicle-Following Model}

The Federal Highway Administration (FHA) of the US Department of transportation defines the driver's reaction time as the PIEV time in its Unified Traffic Control Device Handbook. PIEV time consists of four parts:

- Perception process: A driver perceives the change in driving environment.

- Intellection process: The driver analyzes the information about the change.

- Evaluation process: The driver determines driving behavior based on his analysis.

- Volition process: The driver executes the driving behavior.

We apply the PIEV process in the vehicle following model and overtaking model. In each time period, we first get the speed and position of each vehicle, calculate the gap, and then determine the driving behavior to continue to follow or change the lane). Depending on the driving behavior, the acceleration is calculated and the speed and position are updated. 
This is mainly based on the behavior of the driver under the current vehicle distance, if the distance $G$ is safe enough, acceleration is feasible; otherwise the vehicles should slow down.

\subsection{Merging model}

The plug car clearance is a time interval that the driver can safely import or through the continuous flow. When more vehicles pass the toll plaza, the driver entering or crossing traffic must be judged from the potential conflict between the vehicle and the own vehicle to make a decision whether to enter or pass through.

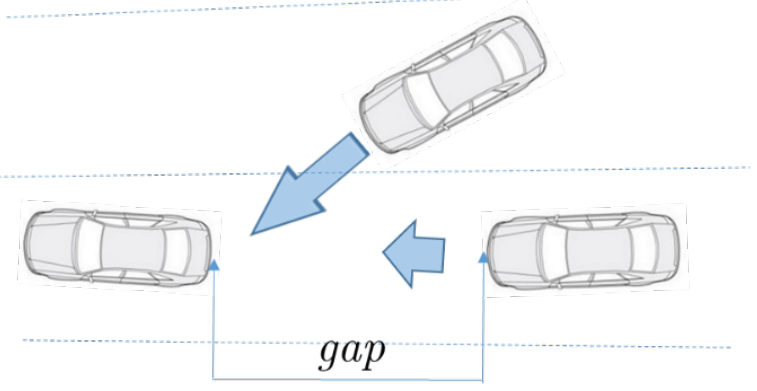

Fig2. Lane change diagram

The probability $P_{0}$ depends on the vehicle, the front vehicle $A$ and the rear vehicle $B$, and $C$ indicates the minimum value of the time slot into which the vehicle enters the vehicle, and $t_{f}$ indicates the vehicle $A$. In the follow-up time between vehicles $B, C$, probability $P_{0}$ satisfies:

$$
P_{0}=\left\{\begin{array}{l}
P_{c}\left(1-0.9 e^{\left(t_{c}-t_{f}\right)}\right) \quad \text { if } t_{c}<t_{f} \\
0.1 \quad \text { if } t_{c} \geq t_{f}
\end{array}\right.
$$

\section{Performance analysis}

According to the above calculation method, we take $L_{0}=50 \mathrm{~m}, \mathrm{D}=112 \mathrm{~m}, \alpha=72^{\circ}, \beta=72^{\circ}, B=8, L=3$. Toll stations takes artificial toll stations. The queuing theory parameters are $\lambda=0.2778$ (Vehicle/s), $\mu=0.0629$ (Vehicle/s) and $\Delta t=1 s$.Using cellular auto or simulation, the results of the indicators are as follows.

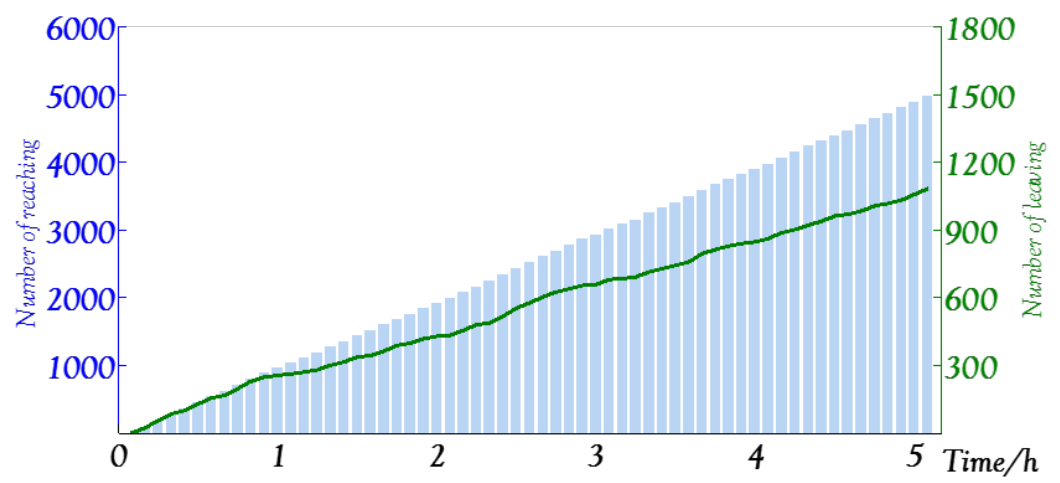

Fig3. The total number of vehicles with time

\begin{tabular}{|c|c|c|c|c|c|c|}
\hline \multirow[b]{2}{*}{$\begin{array}{l}\text { Throughput } \\
\text { (Vehicle/h) }\end{array}$} & \multicolumn{2}{|c|}{ Efficiency index } & \multicolumn{2}{|c|}{ Danger index } & \multirow[b]{2}{*}{$\begin{array}{l}\text { area } \\
(\mathrm{m} 2)\end{array}$} & \multirow[b]{2}{*}{ Cost } \\
\hline & $\begin{array}{c}\text { density } \\
\text { ( Vehicle/m2) }\end{array}$ & $\begin{array}{c}\text { average } \\
\text { velocity } \\
(\mathrm{m} / \mathrm{s})\end{array}$ & $\begin{array}{l}\text { average } \\
\text { vehicle } \\
\text { distance } \\
\quad(\mathrm{m})\end{array}$ & $\begin{array}{l}\text { the } \\
\text { number of } \\
\text { lane } \\
\text { changes } \\
\text { per hour }\end{array}$ & & \\
\hline 205.25 & 0.0065 & 9.89 & 58.13 & 1065.32 & 29462.97 & $29462.97 C$ \\
\hline
\end{tabular}

Table1. The results of the indicators value

It can be seen that the number of vehicles entering the toll station and the toll station is gradually 
increasing with the increase of the time when the size of the toll plaza is $25000 \mathrm{~m}^{2}$, throughput of about 205 per hour, traffic density of about 0.0044 (vehicle $/ \mathrm{m}^{2}$ ), the average speed is $31 \mathrm{~m} / \mathrm{s}$, the average car distance is about $55 \mathrm{~m}$ and the total number of lane changes per hour is 851 times.

In the original model, we assume all toll booth types are conventional (human-staffed) tollbooths. Next, we modify the number and type of toll booths in the CA model, increasing the number of exact-change (automated) tollbooths, and electronic toll collection booths, and we can get the following graphs about the throughput.

\section{The design of the existing charge area}

The gradual transition from the toll plaza to the conventional motor vehicle lane requires that the vehicle travel smoothly. Therefore, the transition section should use the appropriate gradient rate. When the toll station and the width of the road is determined, the longer the transition length, the smaller the gradient, the more natural vehicles from the toll plaza into the conventional motor vehicle lanes. Linear transition should be smooth, round, to avoid that the traffic path is too reluctant, at the same time we should pay attention to construction difficulty.

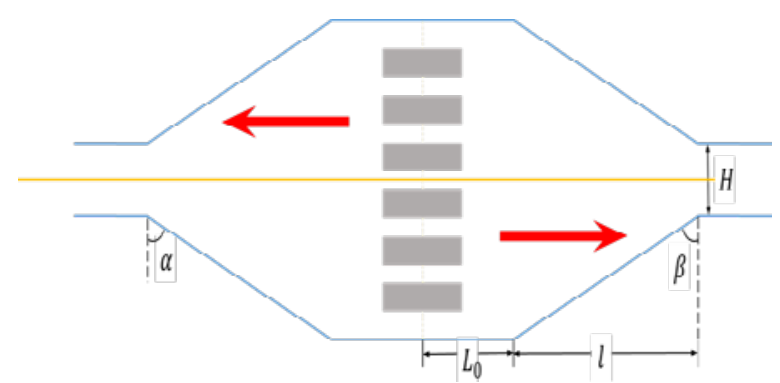

Fig4. Schematic diagram of toll plaza

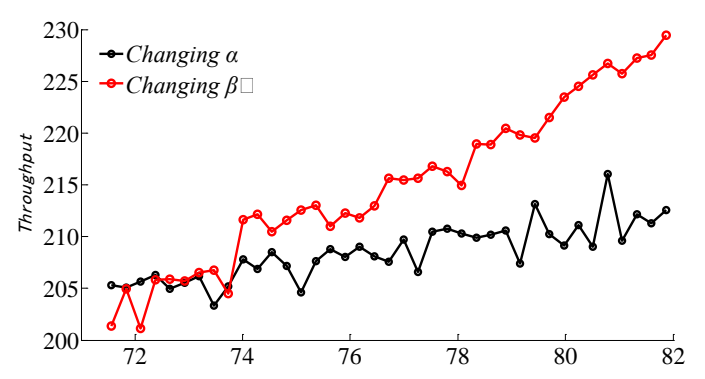

Fig5. Variation of throughput with $\alpha$ and $\beta$

It can be seen from the figure that with the increase of the angle, the throughput curve fluctuates up and down, but the overall trend is increasing. The change range of $\beta$ is obviously bigger than the variation range of $\alpha$. When $\alpha$ increases, the increase of the toll plaza entrance area makes more vehicles to enter. The early vehicles less, the throughput has increased significantly but the number of toll stations must be fixed, late toll station efficiency and stability, throughput is also basically stable. When $\beta$ increases, the increase in the area of the toll plaza's exports allowed the vehicles to have more lanes to choose from, without waiting in line for time. So, throughput was a clear upward trend.

\section{Conclusions}

By changing the area of entrance and exit, we can draw a conclusion that: safety and efficiency are always negatively related to costs. With the increase of area, the throughput and Efficiency Index will increase at the same time while Danger Index decreases by contraries. When it comes to electronic toll collection booths (ETC) or exact-change tollbooths (EC), the throughput will increase on account of the shrink of service time. But when the total flow exiting from the toll booths exceeds the capacity of the downstream highway, fan-shaped area will be congested due to capacity drop. Based on the simulation results, the proper proportions of ETC and EC should vary from 1/3 to 1/2.

\section{References}

[1] Albert, Schaufler. Toll plaza design [M]. U.S.A: Transportation Research Board, 1997:8-40

[2] Nico M, van Dijk, Mark D. Designing the Wester scheldetunnel Toll Plaza Using a Combination of Queueing and Simulation [C]. Proceeding of the 1999 Winter Simulation Conference, 1999: 1272-1279

[3] Pratelli, Schoen. Optimal design of motorway toll stations [J]. European Trans-port Conference, 2003(9), 8:1-33 